$\xi=-1$ 囬

\title{
The Beauty Industry and Engineering Technologies
}

\author{
Hye-Jin Kwon ${ }^{1} *$

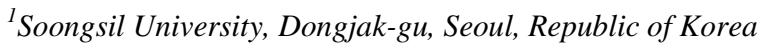 \\ *Corresponding author E-mail:kwonhj0070@ssu.ac.kr
}

\begin{abstract}
This study attempted to converge beauty and engineering for the purpose of overcoming the limitations of the current emotion-centered beauty industry and creating quantitative and objective values of beauty. This study also examined engineering technologies currently in use in the cosmetics field and analyzed them in a quantitative manner. Thereby this study intends to provide basic knowledge on a new convergent study area that is broadly applicable to the beauty industry and its all related fields.
\end{abstract}

Keywords: Beauty engineering, Beauty industry, Cosmetics, Beauty convergence, Objective values of beauty

\section{Introduction}

Beauty engineering is a study area of new concept where beauty and engineering are converged so as to overcome the limitations of existing, subjective, emotion- and experience-centered beauty industry and to create quantitative, systematic, and engineering values of beauty. There is no clear and obvious definition of beauty engineering yet, both in and out of Korea. This study grafted the transformation system of chemical engineering into the beauty area for the purpose of recreating the values of beauty that are measurable and can develop and evolve based on the high potential and abundant experience of the Korean beauty (K-beauty) industry.

Improved living standard and population aging led to an increase of demand for goods for a beautiful life as well as health. As of 2017 , the global beauty industry witnessed rapid growth with its market size at 265 billion dollars [1][2]. In the global beauty market where the United States, Japan, and Europe Union rank among the top 10 countries, K-beauty market has made rapid growth and ranks the $11^{\text {th }}$. Besides, the domestic beauty market has seen a high annual average growth rate of $10 \%$. Therefore, there is an urgent need to heighten the value of Korean beauty industry and strengthen its international competitiveness and to develop Korean original source technologies and secure excellent professional manpower.

Beauty engineering will help Korea present itself as a beauty mecca in the global beauty market and create related convergence areas and new convergence industries based on comprehensive concepts such as the quantification of beauty values, development of core components for beauty, alternative clinical engineering, vital reaction engineering, international standardization, future life industry, and new wellbeing-oriented industry [3][4]. Accordingly, this study intends to provide basic knowledge for the new area of convergence study that is widely applicable to medicine, public health, culture, arts, life, and other industries as well as the beauty industry by connecting beauty with engineering based on such concept of beauty engineering.

\section{Engineering Technologies Related with the Beauty Cosmetics Industry}

\subsection{Development of Natural Antioxidant Materials}

As a new paradigm has recently emerged that development of health products, natural cosmetics, and pharmaceutical products from natural resources is the best way to guarantee people's health in the future, much attention has been given to natural cosmetics materials [5]. In particular, the problems with synthetic materials emerged and most materials sold in the Korean cosmetics market are imported; developing cosmetics materials using native plants is an essential need. In general, development of natural materials involves excavating natural components as well as natural substances of medicinal plants used in the traditional herbal medicine. Research on natural plants begins with collection of plants and extraction of active constituents. A precise method to extract them depends on the part of plants to be used, their moisture content, and materials to be extracted [6]. How to separate and refine active materials should differ according to the physical and chemical characteristics of the given material to separate. Among separation and refinement methods that are based on various principles, appropriate methods should be applied in an effective manner. In general, methods used in the study area of natural substances are slightly different from refinement methods in organic chemistry. It is most common to track certain framework compounds, that is, compounds of certain groups [7]. Polyphenol and flavonoid, representative compounds contained in a wide variety of plants, protect the human body through their functions as an antioxidant that inhibits the formation of free radicals resulting from oxidative stress, as well as the functions of suppressing reactions to break up free radicals and of recovering damaged parts. Representative methods to detect such components in plants include high performance liquid chromatography (HPLC), oxygen radical absorbance capacity (ORAC), and oxidative radical de-colorization. They are widely in use as methods to quantify bioactive substances in cosmetics materials. Fig. 1 shows the most widely used methods to measure anti-oxidative activity of plants. 


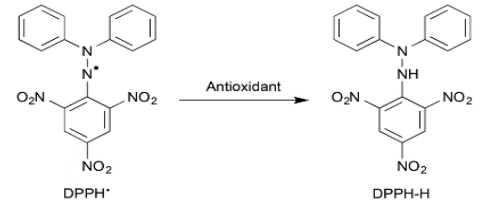

(a)

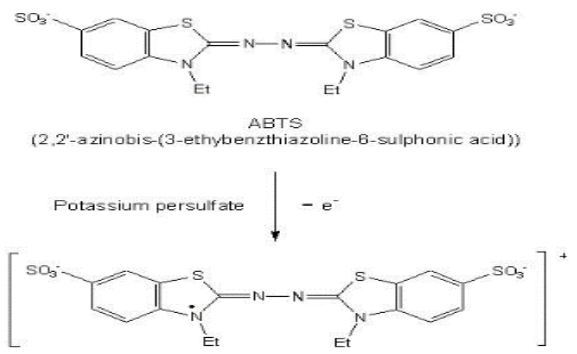

(b)

Fig. 1: Measure anti-oxidative activity. (a)DPPH assay (b) ABTS assay [3]

\subsection{Fermentation Process}

The fermentation method is the oldest in history and has been applied to diverse areas such as food, medicine, and cosmetics. During fermentation of lactic acid, acetic acid, alcohol, etc., useful components and nutrients are increased by the action of microorganisms. In addition, fermentation of materials abundant in proteins, essential amino acids, and vitamins destroys toxins and increases production of bioactive substances. Recently, research on fermentation of natural bioactive substances using microorganisms has been actively conducted [8]. Fermentation of natural substances using microorganisms not only maintains most nutrients intact, excluding the energy sources of microorganisms but also liberates all kinds of hydrolytic enzymes secreted by microorganisms and bioactive substances combined with intracellular tissues, thereby greatly heightening their bioavailability rate. After all, fermentation increases absorption of organic matters by breaking the molecular structure of organic matters by microorganisms and produces abundant nutrients by generating new materials more excellent than original organic matters in the process. The process also facilitates absorption by the skin through the smaller molecular structure of the products resulting from fermentation because they are consumed and excreted by microorganisms. Such advantages of fermentation led to much research aimed at examining physiological activity of medical herbs and extracts from edible plants through heat treatment and maturation and fermentation. With reports on anti-oxidative effects of fermented extracts, active research has been recently conducted on skin aging.

\subsection{Antibacterial Experiments for Cosmetics Quality Control}

When microorganisms are contaminated and proliferate in cosmetics, resulting changes in color, smell, and viscosity, and their decomposed components may provide factors that degrade the value of the products such as skin irritation and the risk of infection with pathogenic microorganisms [9]. Therefore, preservatives should be added to prevent microorganism contamination. When selecting preservatives to be added to a new product, the possibility of contamination by various kinds of microorganisms and resistant bacteria and factors that inhibit preservative action should be taken into account; using several preservatives at the same time is more beneficial than using a single preservative. Therefore, to prevent inhibition action on preservatives by other mixed ingredients while improving antimicrobial action of preservatives, preservatives with synergetic effects should be used in combination, which may decrease the amount of preservatives used and skin irritation. Major microorganisms used to test cosmetic products' preservative capacity include Staphylococcus aereus, a gram- positive bacterium, and Escherichia coil and Pseudomonas aeruginosa, gram-negative bacteria.

\section{Engineering Technologies Related with the Beauty Service Industry}

\subsection{Skin Analysis Methods Used in the Skin Care In- dustry}

The market size of the Korean beauty service industry and skin care industry is annually growing. Despite global recession, those industries expect that annual sales will increase. The reason is that many people are socially aware of their image as well as their health and appearance [10]. This increased demands for specialized beauty services. Thus far, with rapid change in social and economic conditions, an increase of income level of customers, and change in awareness about beauty, the management concept of skin care shops has gone through changes; ways to satisfy customers' diverse needs and impress them have been pursued. Away from management marketing that merely seeks to sell technologies and provide services, management of continuous relationship with customers should be considered important so that customer values may be maximized by converting one-time customers to lifetime customers. In other words, customers' service desires increased due to provision of diverse services should be satisfied; more specialized and scientific methods to meet their desires are required. A precise skin analysis method customized to such customers' needs may elicit trust from them. Applying proper skin analysis methods will help satisfy customers' tastes and increase sales by analyzing data on oil and moisture levels of skin, pigments, wrinkles, and elasticity in a scientific and objective manner.

\subsection{Brain-Wave Analysis in Use in the Emotional Con- sumption Industry}

With the advent of the $20^{\text {th }}$ century, the beauty industry attempted to connect emotional aspects with technological development in order to adapt to the trends of the era like LOHAS (lifestyles of health and sustainability). In the 21the century, new industries such as "incense-burner marketing" that increases consumers' desire to purchase products using their emotions and psychology has been rapidly emerging. Diverse research aimed at providing the scientific bases on the effects of human emotions and stress that are affected by incenses on their physiological capacity has been conducted [10].

Brainwave physiology to examine the basic mechanism where 'such incenses' affect the human body is drawing attention. It may access and analyze brain conditions such as brain awakening, activation, state of anxiety, and state of stability in response to stimuli using brainwaves to quantitatively measure the brain's activation states. Further, if consumers' emotions are analyzed through research on techniques of data construction and analysis based on various brainwave indices obtained from consumers by quantitatively analyzing brain responses to Munsell's 10 hues and makeup colors, the result may be usefully employed in diverse areas such as image display, psychotherapy, design marketing, and emotional engineering-based beauty treatment [11]. This is a method to quantitatively analyze changes in brainwave values according to color stimuli using diverse brainwave indices and closely examine correlation between colors and brainwaves. Such method may be diversely applied to the emotional consumption industry by quantitatively analyzing the images of colors through brainwaves. 


\subsection{Color Marketing Used in the Emotional Consump- tion Industry}

Color marketing aims to sell products using design and color Colors are the most quickly perceived objects through vision among the five senses, make up a large portion in the formation of images, and are differently perceived by individuals. A product's colors represent its producer's images and are one of the factors directly related with sales [12]. Colors are one of the important elements of aestheticization by stimulating sensitivity through changes in perception by vision. When consumers choose a product, they generally compare it with other products having similar characteristics such as performance and price. However, they do not always make planned purchases. In many cases, they purchase what they like regardless of its usage or their purchase plan. Consumers do not select goods solely based on rational and reasonable standards [13]. They want to obtain psychological and mental satisfaction by purchasing goods. It is very crucial to establish marketing strategies in line with such changes in consumers. Cosmetics containers bring into the images of products relief using colors and should be able to stimulate consumers' desire to purchase through visual satisfaction. The colors of cosmetics containers should unconsciously reach consumers' mind and should be readily accessible to purchasers by drawing attention from them and giving pleasure. As part of such color marketing strategies, statistical data on the production costs of all cosmetics including fundamental cosmetics may be used to predict consumer demand. In addition, it is possible to identify consumers' consumption types by examining the color trend of cosmetics containers produced in Korea and analyzing correlation between production cost and colors of cosmetics [11]. Thereby, based on the data on the current status analyzed in consideration of tangible aspects revealed by correlation between cosmetics production cost and containers' color elements, colors may be understood and the predicted color trend may be proposed. Verifying whether the color trend derived through empirical analysis of and experiments on consumers may satisfy actual consumers' purchase behaviors and desires and whether the color marketing strategies are proper will help establish cosmetics marketing strategies that stimulate consumers' emotions.

\section{Engineering Technologies Associated with the Beauty-Related Industries}

\subsection{Body Shape Analysis Used in the Beauty Health Industry}

Intertwined with increasing lookism, a rise in the obese population, and the wellbeing trend, body shape management services provided by the beauty industry have become segmentized, specialized, and aggrandized, which has led to the rapid growth of the relevant health industry [14]. Accordingly, academic research on body shape management services should be performed and diverse methods to activate the services should be developed. Empirical analysis on body shape management shops, whose market size recently began to grow, can closely examine the factors that affect customers' perception about their service quality [11] and identify relationship between their association with customer satisfaction and reuse rate, thereby widening the theoretical scope of service quality and customer satisfaction and presenting the direction of marketing strategies for specialized body shape management shops and the direction of service quality improvement and profit creation as a specialized business.

\subsection{Nutrition Instruction and Development of Exercise Programs for Use by the Beauty Health Industry}

People in modern society are leading the most abundant life throughout the human history, as the development of science and medicine and diverse foods supplied in large quantities resulting from epoch-making development of food processing technologies has led to the improvements in their nutritional conditions and diet, thereby enhancing the quality of life. The development of food processing technologies changed humans' dietary life; they are exposed, rather than natural foods, to fast foods and instant foods high in calories, fats, proteins, carbohydrates, and salt but with insufficient vitamins, minerals, and fibers [15]. Obesity and all kinds of metabolic diseases have become the biggest factor that threatens human health across the world. As such, obesity may be not detrimental to human health only when it is prescribed, managed, and treated as a disease. Dietary and exercise therapies that recommend nutritionally BLCD (balanced low calorie diet) among the methods to manage obesity is the safest method that does not pose the risk of side effects that may occur in medication or surgical treatment. Exercise facilitates basal metabolism and energy consumption, decreasing weight and body fat as well as enhancing physical strength; exercise is presented as an effective method to treat obesity. Therefore, for safe and efficient obesity prevention and management, precise analysis of individual conditions and effective programs, in addition to mere exercise and diet management, should be developed to satisfy customers' needs for the purpose of reducing socio-economic costs incurred to treat obesity. To this end, relevant quantitative and systematic research is necessary

\section{Conclusion}

Beauty engineering is a new paradigm of quantifying the values of beauty based on human emotions. Securing original source technologies is made possible by developing a diversity of core materials such as bio-natural substances as well as eco-friendly, nanocomposite, and bio-mimetic materials beyond materials that provide aesthetic values only. Amid the surging demand for cosmeceuticals and functional beauty products, national competitiveness should be strengthened through continuous implementation of product standardization and quantification as well as international standardization. Moreover, education and research on devices and systems for analysis on and quantification of product elements are also in an urgent need. It is considered that the engineering concept presented in this study may provide basic data for the re-creation of the values of beauty.

\section{Acknowledgement}

This research was supported by 2018 Basic Research Program through the National Research Foundation of Korea funded by the Education, Science and Technology. (No.2018008274)

\section{References}

[1] Asia http://www asiatoday co.kr

[2] Hankyung 016.1 .19 http://bntnews.hankyung.com/apps/news?popup $=0 \&$ nid $=03 \& \mathrm{c} 1=03$ $\& c 2=03 \& c 3=00 \&$ nkey $=201305201925593 \&$ mode $=$ sub_view

[3] Ikuo N, Kei O, Yukihiro O, Ken M, Toshihiko O, Shunichi F (2016), Aluminium ion-promoted radical-scavenging reaction of methylated hydroquinone derivatives. Org. Biomol. Chem. 14, 7956-7961

[4] Cho HK, Lee JY, Seo WT, Kim MK, Cho KM (2012), Quality characteristics and antioxidant effects during Makgeolli fermentation by purple sweet potato-rice nuruk. Korean J Food Sci Technol 44, 728-735. 
[5] Wang SJ, Lee HJ, Cho JY, Park KH, Moon JH (2012), Isolation and Identification of Antioxidants from Makgeolli. Korean J Food Sci Technol 44. 14-20.

[6] Jeon HJ, Kwon HJ (2016), Anti-inflammation Effect of Gynura Procumbens extract. Journal of Digital Convergence 14. 515-520.

[7] Park SH, Cha KO (2016), Oriental and Western Food Effects Analysis of Misutgaru for Fusion Remedy in Diabetes Mellitus. Journal of the Korea Convergence Society 7. 137-143.

[8] Seo GU, Choi SY, Kim TW, Ryu SG, Park JH, Lee SC (2013), Functional activities of Makgeolli by-produccts as cosmetic materials. J Korean Soc Food Sci Nut 42. 505-511.

[9] Park JY, Jin JS, Kang HY, Jeong EH, Lee JC, Lee YC, Seol SY, Cho DT, Kim J (2007), A comparison of adult and pediatric methicillin-resistant Staphylococcus aureus isolates collected from patients at a university hospital. J. of the Microbiol 45. 447-452.

[10] Lee YJ, Kim JH (2017), A Study of Consumer's Emotional Response on Musical Stimulus through EEG Analysis : Based on Color Perception of Consumers. Korean Journal of Business Administration 30. 2147-2172.

[11] Oh HK (2018), Survey of clothing habits in female senior citizens according to subjective physical changes and health status. J. Korea Soc. Beauty Art 19. 77-93.

[12] Park JS, Kwon HJ (2017), Perception about Makeup Influence on Man's Makeup and Their Success. Journal of the Korea Convergence Society 8. 231-237.

[13] Lee JY (2015), The Analysis of Skin Care Service Marketing-Mix Factor of Skin Care Shops Satisfaction and Loyalty. Kor. J. Aesthet. Cosmetol 13. 873-881.

[14] Kang HJ, Kim GP, Cho YH (2017), A Study on the Relationship of Skin Surface $\mathrm{pH}$ with Nutrient Intake or Dietary Pattern in Healthy Adults. The Korean Journal of Food And Nutrition 30. 9-18.

[15] Lee DS, Shon JH, Yoo HS (2018), Case Study of Chronic Exercise Effect on Facial Skin Condition and Subjective Well-being. Korean Journal of Sport Psychology 29. 41-51 